Supplement of Nat. Hazards Earth Syst. Sci., 19, 1119-1127, 2019

https://doi.org/10.5194/nhess-19-1119-2019-supplement

(c) Author(s) 2019. This work is distributed under

the Creative Commons Attribution 4.0 License.

(c) (1)

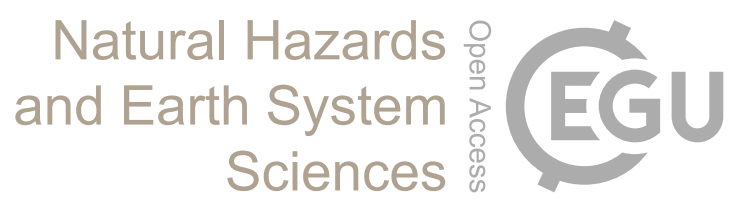

Supplement of

\title{
Taylor's power law in the Wenchuan earthquake sequence with fluctua- tion scaling
}

\section{Peijian Shi et al.}

Correspondence to: Mei Li (mei_seis@163.com) and Yang Li (yangli@d.umn.edu)

The copyright of individual parts of the supplement might differ from the CC BY 4.0 License. 


\section{Supplementary tables}

Table S1. Fitted results of two linear equations for different block sizes A (time interval in days) from 2008-2016

\begin{tabular}{|c|c|c|c|c|c|c|c|c|c|c|c|c|}
\hline \multirow{3}{*}{$\frac{A}{4}$} & \multicolumn{8}{|c|}{ Equation $\log _{10}($ variance $)=c+b \log _{10}($ mean $)$} & \multicolumn{4}{|c|}{ Equation $\log _{10}($ variance $)=d+2 \log _{10}($ mean $)$} \\
\hline & \multirow{2}{*}{$\frac{\hat{c}}{0.016}$} & \multicolumn{2}{|c|}{$95 \% \mathrm{CI}$ of $\hat{c}$} & \multirow{2}{*}{$\frac{\hat{b}}{2.080}$} & \multicolumn{2}{|c|}{$95 \%$ CI of $\hat{b}$} & \multirow{2}{*}{$\frac{R^{2}}{0.9670}$} & \multirow{2}{*}{$\frac{\mathrm{RMSE}_{1}}{0.4083}$} & \multirow{2}{*}{$\frac{\hat{d}}{1.263}$} & \multicolumn{2}{|c|}{$95 \%$ CI of $\hat{d}$} & \multirow{2}{*}{$\frac{\mathrm{RMSE}_{2}}{0.4170}$} \\
\hline & & -0.404 & 0.435 & & 2.053 & 2.107 & & & & 1.234 & 1.292 & \\
\hline 5 & 0.034 & -0.450 & 0.518 & 2.085 & 2.054 & 2.116 & 0.9654 & 0.4180 & 1.359 & 1.325 & 1.392 & 0.4276 \\
\hline 7 & 0.285 & -0.270 & 0.839 & 2.077 & 2.041 & 2.112 & 0.9677 & 0.4016 & 1.489 & 1.451 & 1.527 & 0.4097 \\
\hline 8 & 0.345 & -0.251 & 0.941 & 2.077 & 2.039 & 2.115 & 0.9676 & 0.3924 & 1.561 & 1.521 & 1.600 & 0.4004 \\
\hline 11 & 0.669 & -0.077 & 1.415 & 2.064 & 2.017 & 2.111 & 0.9634 & 0.4135 & 1.683 & 1.634 & 1.732 & 0.4187 \\
\hline 12 & 0.526 & -0.234 & 1.286 & 2.075 & 2.027 & 2.123 & 0.9656 & 0.3976 & 1.714 & 1.665 & 1.763 & 0.4049 \\
\hline 13 & 0.725 & -0.099 & 1.548 & 2.066 & 2.014 & 2.117 & 0.9626 & 0.4097 & 1.767 & 1.715 & 1.820 & 0.4150 \\
\hline 14 & 0.533 & -0.290 & 1.356 & 2.079 & 2.027 & 2.131 & 0.9657 & 0.3979 & 1.788 & 1.735 & 1.842 & 0.4059 \\
\hline 15 & 0.647 & -0.242 & 1.537 & 2.073 & 2.017 & 2.128 & 0.9626 & 0.4145 & 1.801 & 1.744 & 1.859 & 0.4210 \\
\hline 16 & 0.780 & -0.147 & 1.707 & 2.066 & 2.008 & 2.125 & 0.9619 & 0.4118 & 1.838 & 1.779 & 1.896 & 0.4172 \\
\hline 20 & 1.057 & 0.027 & 2.087 & 2.055 & 1.991 & 2.120 & 0.9625 & 0.4008 & 1.943 & 1.879 & 2.007 & 0.4046 \\
\hline 21 & 0.982 & -0.018 & 1.982 & 2.061 & 1.998 & 2.123 & 0.9663 & 0.3853 & 1.951 & 1.888 & 2.015 & 0.3901 \\
\hline 22 & 0.875 & -0.244 & 1.994 & 2.068 & 1.998 & 2.138 & 0.9605 & 0.4149 & 1.964 & 1.895 & 2.034 & 0.4203 \\
\hline 23 & 0.728 & -0.345 & 1.802 & 2.078 & 2.011 & 2.145 & 0.9654 & 0.3959 & 1.979 & 1.911 & 2.048 & 0.4036 \\
\hline
\end{tabular}




\begin{tabular}{|c|c|c|c|c|c|c|c|c|c|c|c|c|}
\hline 24 & 0.829 & -0.315 & 1.974 & 2.073 & 2.001 & 2.144 & 0.9624 & 0.4043 & 1.993 & 1.922 & 2.065 & 0.4106 \\
\hline 25 & 0.749 & -0.418 & 1.917 & 2.079 & 2.006 & 2.152 & 0.9626 & 0.4127 & 2.012 & 1.938 & 2.086 & 0.4203 \\
\hline 26 & 0.898 & -0.303 & 2.098 & 2.072 & 1.998 & 2.147 & 0.9621 & 0.4055 & 2.057 & 1.982 & 2.131 & 0.4117 \\
\hline 27 & 1.028 & -0.069 & 2.124 & 2.064 & 1.996 & 2.133 & 0.9692 & 0.3696 & 2.059 & 1.990 & 2.128 & 0.3752 \\
\hline 28 & 0.844 & -0.309 & 1.996 & 2.078 & 2.006 & 2.149 & 0.9679 & 0.3813 & 2.096 & 2.022 & 2.169 & 0.3893 \\
\hline 29 & 0.733 & -0.566 & 2.033 & 2.083 & 2.002 & 2.164 & 0.9610 & 0.4132 & 2.069 & 1.988 & 2.149 & 0.4212 \\
\hline 30 & 1.111 & -0.169 & 2.391 & 2.061 & 1.981 & 2.140 & 0.9624 & 0.3973 & 2.089 & 2.010 & 2.167 & 0.4018 \\
\hline 31 & 1.401 & 0.043 & 2.760 & 2.045 & 1.961 & 2.129 & 0.9590 & 0.4096 & 2.125 & 2.043 & 2.207 & 0.4119 \\
\hline 32 & 0.951 & -0.385 & 2.287 & 2.072 & 1.989 & 2.155 & 0.9623 & 0.4045 & 2.100 & 2.017 & 2.183 & 0.4107 \\
\hline 33 & 1.401 & 0.116 & 2.686 & 2.044 & 1.964 & 2.123 & 0.9653 & 0.3838 & 2.102 & 2.023 & 2.181 & 0.3862 \\
\hline 34 & 1.367 & -0.041 & 2.775 & 2.047 & 1.96 & 2.135 & 0.9601 & 0.4068 & 2.131 & 2.046 & 2.216 & 0.4095 \\
\hline 35 & 1.171 & -0.221 & 2.562 & 2.061 & 1.975 & 2.147 & 0.9624 & 0.4017 & 2.152 & 2.067 & 2.238 & 0.4062 \\
\hline 36 & 1.185 & -0.174 & 2.543 & 2.061 & 1.976 & 2.145 & 0.9653 & 0.3853 & 2.159 & 2.075 & 2.243 & 0.3899 \\
\hline 37 & 1.175 & -0.203 & 2.552 & 2.063 & 1.978 & 2.148 & 0.9654 & 0.3881 & 2.189 & 2.104 & 2.275 & 0.3931 \\
\hline 38 & 0.881 & -0.553 & 2.316 & 2.082 & 1.993 & 2.17 & 0.9641 & 0.3981 & 2.198 & 2.109 & 2.288 & 0.4062 \\
\hline 39 & 1.230 & -0.045 & 2.504 & 2.062 & 1.983 & 2.141 & 0.9721 & 0.3492 & 2.228 & 2.149 & 2.308 & 0.3546 \\
\hline 40 & 1.197 & -0.057 & 2.451 & 2.064 & 1.986 & 2.141 & 0.9737 & 0.3392 & 2.229 & 2.151 & 2.307 & 0.3451 \\
\hline 41 & 0.855 & -0.734 & 2.443 & 2.083 & 1.984 & 2.181 & 0.9601 & 0.4200 & 2.190 & 2.091 & 2.288 & 0.4279 \\
\hline 42 & 1.437 & -0.004 & 2.878 & 2.049 & 1.960 & 2.138 & 0.9664 & 0.3780 & 2.231 & 2.142 & 2.319 & 0.3811 \\
\hline 43 & 1.213 & -0.302 & 2.729 & 2.064 & 1.970 & 2.157 & 0.9645 & 0.3831 & 2.243 & 2.152 & 2.335 & 0.3880 \\
\hline 44 & 1.586 & -0.064 & 3.235 & 2.041 & 1.939 & 2.143 & 0.9585 & 0.4068 & 2.255 & 2.157 & 2.352 & 0.4087 \\
\hline 45 & 1.789 & 0.227 & 3.351 & 2.029 & 1.932 & 2.126 & 0.9627 & 0.3875 & 2.259 & 2.165 & 2.352 & 0.3886 \\
\hline 46 & 0.929 & -0.527 & 2.384 & 2.081 & 1.991 & 2.171 & 0.9700 & 0.3678 & 2.235 & 2.143 & 2.327 & 0.3767 \\
\hline 47 & 0.738 & -0.804 & 2.28 & 2.094 & 1.999 & 2.189 & 0.9674 & 0.3760 & 2.259 & 2.164 & 2.354 & 0.3871 \\
\hline 48 & 1.043 & -0.612 & 2.699 & 2.076 & 1.973 & 2.178 & 0.9631 & 0.4000 & 2.265 & 2.164 & 2.367 & 0.4068 \\
\hline 49 & 1.694 & -0.022 & 3.409 & 2.036 & 1.931 & 2.142 & 0.9597 & 0.4023 & 2.284 & 2.182 & 2.385 & 0.4038 \\
\hline
\end{tabular}




\begin{tabular}{|c|c|c|c|c|c|c|c|c|c|c|c|c|}
\hline 50 & 1.127 & -0.538 & 2.792 & 2.073 & 1.970 & 2.176 & 0.9639 & 0.3913 & 2.307 & 2.206 & 2.408 & 0.3977 \\
\hline 51 & 1.343 & -0.349 & 3.035 & 2.061 & 1.957 & 2.166 & 0.9637 & 0.3851 & 2.337 & 2.237 & 2.438 & 0.3896 \\
\hline 52 & 1.218 & -0.433 & 2.868 & 2.066 & 1.964 & 2.168 & 0.9660 & 0.3757 & 2.289 & 2.190 & 2.388 & 0.3812 \\
\hline 53 & 1.859 & 0.173 & 3.544 & 2.030 & 1.926 & 2.134 & 0.9641 & 0.3770 & 2.346 & 2.247 & 2.446 & 0.3781 \\
\hline 54 & 0.970 & -0.778 & 2.718 & 2.081 & 1.973 & 2.189 & 0.9638 & 0.4051 & 2.278 & 2.169 & 2.388 & 0.4132 \\
\hline 55 & 1.464 & -0.330 & 3.258 & 2.054 & 1.943 & 2.164 & 0.9618 & 0.3963 & 2.338 & 2.231 & 2.445 & 0.3998 \\
\hline 56 & 1.748 & 0.070 & 3.427 & 2.039 & 1.936 & 2.142 & 0.9667 & 0.3618 & 2.380 & 2.282 & 2.478 & 0.3637 \\
\hline 57 & 1.597 & -0.155 & 3.348 & 2.046 & 1.938 & 2.154 & 0.9647 & 0.3779 & 2.344 & 2.240 & 2.448 & 0.3806 \\
\hline 58 & 1.115 & -0.726 & 2.956 & 2.076 & 1.963 & 2.19 & 0.9629 & 0.3984 & 2.349 & 2.237 & 2.461 & 0.4053 \\
\hline 59 & 2.030 & 0.258 & 3.802 & 2.022 & 1.914 & 2.131 & 0.9646 & 0.3735 & 2.395 & 2.291 & 2.499 & 0.3741 \\
\hline 60 & 1.817 & 0.094 & 3.541 & 2.035 & 1.929 & 2.141 & 0.9675 & 0.3615 & 2.387 & 2.285 & 2.489 & 0.3631 \\
\hline 61 & 1.691 & -0.026 & 3.409 & 2.042 & 1.937 & 2.148 & 0.9685 & 0.3579 & 2.379 & 2.277 & 2.482 & 0.3602 \\
\hline 62 & 1.985 & 0.138 & 3.833 & 2.025 & 1.912 & 2.139 & 0.9640 & 0.3717 & 2.396 & 2.289 & 2.503 & 0.3725 \\
\hline 63 & 1.700 & -0.286 & 3.686 & 2.040 & 1.918 & 2.163 & 0.9590 & 0.4010 & 2.354 & 2.238 & 2.469 & 0.4028 \\
\hline 64 & 2.255 & 0.385 & 4.125 & 2.009 & 1.894 & 2.124 & 0.9633 & 0.3746 & 2.401 & 2.292 & 2.509 & 0.3747 \\
\hline 65 & 2.174 & 0.319 & 4.029 & 2.013 & 1.899 & 2.127 & 0.9648 & 0.3719 & 2.385 & 2.276 & 2.494 & 0.3721 \\
\hline 66 & 2.058 & 0.213 & 3.904 & 2.018 & 1.905 & 2.132 & 0.9661 & 0.3701 & 2.353 & 2.243 & 2.462 & 0.3706 \\
\hline 67 & 2.410 & 0.479 & 4.34 & 1.999 & 1.880 & 2.118 & 0.9623 & 0.3747 & 2.398 & 2.286 & 2.509 & 0.3747 \\
\hline 68 & 1.932 & -0.025 & 3.888 & 2.028 & 1.908 & 2.149 & 0.9633 & 0.3772 & 2.391 & 2.278 & 2.505 & 0.3781 \\
\hline 69 & 1.773 & -0.301 & 3.847 & 2.042 & 1.914 & 2.169 & 0.9605 & 0.3957 & 2.450 & 2.329 & 2.571 & 0.3976 \\
\hline 70 & 2.379 & 0.241 & 4.517 & 2.004 & 1.872 & 2.135 & 0.9565 & 0.3993 & 2.440 & 2.318 & 2.561 & 0.3993 \\
\hline 71 & 1.169 & -0.714 & 3.052 & 2.074 & 1.958 & 2.190 & 0.9687 & 0.3717 & 2.363 & 2.247 & 2.480 & 0.3789 \\
\hline 72 & 2.381 & 0.400 & 4.363 & 2.003 & 1.882 & 2.125 & 0.9643 & 0.3661 & 2.433 & 2.319 & 2.547 & 0.3661 \\
\hline 73 & 2.194 & 0.319 & 4.070 & 2.014 & 1.899 & 2.129 & 0.9681 & 0.3551 & 2.423 & 2.312 & 2.534 & 0.3553 \\
\hline 74 & 2.322 & 0.484 & 4.161 & 2.006 & 1.893 & 2.119 & 0.9699 & 0.3414 & 2.422 & 2.314 & 2.529 & 0.3415 \\
\hline 75 & 1.807 & -0.255 & 3.87 & 2.039 & 1.913 & 2.166 & 0.9636 & 0.3826 & 2.448 & 2.327 & 2.569 & 0.3845 \\
\hline
\end{tabular}




\begin{tabular}{|c|c|c|c|c|c|c|c|c|c|c|c|c|}
\hline 76 & 2.332 & 0.340 & 4.324 & 2.01 & 1.888 & 2.133 & 0.9660 & 0.3566 & 2.501 & 2.387 & 2.615 & 0.3568 \\
\hline 77 & 2.128 & -0.029 & 4.285 & 2.021 & 1.888 & 2.153 & 0.9618 & 0.3834 & 2.466 & 2.341 & 2.59 & 0.3840 \\
\hline 78 & 2.325 & 0.269 & 4.381 & 2.009 & 1.883 & 2.135 & 0.9647 & 0.3608 & 2.468 & 2.351 & 2.585 & 0.3609 \\
\hline 79 & 1.708 & -0.270 & 3.685 & 2.044 & 1.922 & 2.165 & 0.9691 & 0.3529 & 2.418 & 2.301 & 2.535 & 0.3554 \\
\hline 80 & 2.304 & 0.146 & 4.462 & 2.011 & 1.879 & 2.144 & 0.9624 & 0.3770 & 2.484 & 2.361 & 2.608 & 0.3772 \\
\hline 81 & 2.428 & 0.248 & 4.608 & 2.002 & 1.868 & 2.136 & 0.9624 & 0.3743 & 2.458 & 2.333 & 2.583 & 0.3743 \\
\hline 82 & 2.371 & 0.133 & 4.608 & 2.007 & 1.870 & 2.144 & 0.9606 & 0.3820 & 2.484 & 2.356 & 2.611 & 0.3821 \\
\hline 83 & 1.727 & -0.370 & 3.824 & 2.045 & 1.916 & 2.174 & 0.9664 & 0.3701 & 2.463 & 2.339 & 2.588 & 0.3728 \\
\hline 84 & 2.479 & 0.176 & 4.783 & 2.003 & 1.862 & 2.144 & 0.9595 & 0.3871 & 2.529 & 2.398 & 2.659 & 0.3871 \\
\hline 85 & 1.651 & -0.623 & 3.926 & 2.051 & 1.912 & 2.191 & 0.9621 & 0.3958 & 2.488 & 2.353 & 2.623 & 0.3990 \\
\hline 86 & 1.612 & -0.503 & 3.727 & 2.053 & 1.923 & 2.183 & 0.9681 & 0.3528 & 2.473 & 2.351 & 2.596 & 0.3563 \\
\hline 87 & 2.106 & -0.041 & 4.252 & 2.026 & 1.894 & 2.158 & 0.9664 & 0.3430 & 2.531 & 2.413 & 2.649 & 0.3438 \\
\hline 88 & 1.670 & -0.597 & 3.937 & 2.051 & 1.912 & 2.19 & 0.9647 & 0.3625 & 2.498 & 2.371 & 2.625 & 0.3656 \\
\hline 89 & 1.985 & -0.095 & 4.064 & 2.031 & 1.903 & 2.159 & 0.9694 & 0.3322 & 2.487 & 2.371 & 2.603 & 0.3334 \\
\hline 90 & 1.335 & -0.885 & 3.555 & 2.071 & 1.934 & 2.207 & 0.9666 & 0.3598 & 2.487 & 2.360 & 2.615 & 0.3658 \\
\hline 91 & 1.530 & -0.905 & 3.964 & 2.061 & 1.911 & 2.210 & 0.9611 & 0.3802 & 2.516 & 2.380 & 2.652 & 0.3842 \\
\hline 92 & 2.199 & -0.110 & 4.509 & 2.022 & 1.880 & 2.163 & 0.9635 & 0.3581 & 2.550 & 2.423 & 2.677 & 0.3586 \\
\hline 93 & 1.875 & -0.519 & 4.268 & 2.042 & 1.895 & 2.189 & 0.9629 & 0.3736 & 2.558 & 2.422 & 2.693 & 0.3756 \\
\hline 94 & 2.186 & -0.291 & 4.663 & 2.024 & 1.872 & 2.176 & 0.9598 & 0.3803 & 2.578 & 2.441 & 2.715 & 0.3810 \\
\hline 95 & 2.994 & 0.551 & 5.437 & 1.974 & 1.824 & 2.124 & 0.9588 & 0.3685 & 2.572 & 2.439 & 2.705 & 0.3692 \\
\hline 96 & 1.673 & -0.871 & 4.216 & 2.051 & 1.895 & 2.207 & 0.9600 & 0.3973 & 2.501 & 2.354 & 2.648 & 0.4002 \\
\hline 97 & 1.990 & -0.451 & 4.43 & 2.031 & 1.881 & 2.181 & 0.9623 & 0.3821 & 2.495 & 2.355 & 2.636 & 0.3833 \\
\hline 98 & 3.249 & 0.679 & 5.818 & 1.959 & 1.801 & 2.116 & 0.9556 & 0.3819 & 2.576 & 2.436 & 2.717 & 0.3838 \\
\hline 99 & 2.125 & -0.416 & 4.666 & 2.025 & 1.87 & 2.181 & 0.9607 & 0.3832 & 2.536 & 2.393 & 2.679 & 0.3839 \\
\hline 100 & 2.126 & -0.241 & 4.492 & 2.026 & 1.881 & 2.171 & 0.9657 & 0.3575 & 2.546 & 2.413 & 2.680 & 0.3583 \\
\hline
\end{tabular}

RMSE represents root-mean-square error; $R^{2}$ represents the coefficient of determination for the first linear equation. 
Table S2. Fitted results using different data range when block size $A=10$ days

\begin{tabular}{|c|c|c|c|c|c|c|c|c|c|c|c|c|}
\hline \multirow{3}{*}{$\frac{\text { Data range }}{2008-2008}$} & \multicolumn{8}{|c|}{ Equation $\log _{10}($ variance $)=c+b \log _{10}($ mean $)$} & \multicolumn{4}{|c|}{ Equation $\log _{10}($ variance $)=d+2 \log _{10}($ mean $)$} \\
\hline & \multirow{2}{*}{$\frac{\hat{c}}{-2.329}$} & \multicolumn{2}{|c|}{$95 \%$ CI of $\hat{c}$} & \multirow{2}{*}{$\frac{\hat{b}}{2.214}$} & \multicolumn{2}{|c|}{$95 \% \mathrm{CI}$ of $\hat{b}$} & \multirow{2}{*}{$\begin{array}{c}R^{2} \\
0.9614\end{array}$} & \multirow{2}{*}{$\frac{\text { RMSE }_{1}}{0.3665}$} & \multirow{2}{*}{$\frac{\hat{d}}{1.477}$} & \multicolumn{2}{|c|}{$95 \%$ CI of $\hat{d}$} & \multirow{2}{*}{$\begin{array}{c}\mathrm{RMSE}_{2} \\
0.4068\end{array}$} \\
\hline & & -5.916 & 1.259 & & 2.012 & 2.415 & & & & 1.297 & 1.657 & \\
\hline $2008-2009$ & -4.784 & -6.699 & -2.869 & 2.352 & 2.241 & 2.462 & 0.9696 & 0.3515 & 1.308 & 1.187 & 1.429 & 0.4602 \\
\hline $2008-2011$ & 1.110 & -0.182 & 2.402 & 2.027 & 1.949 & 2.106 & 0.9529 & 0.4704 & 1.562 & 1.481 & 1.644 & 0.4713 \\
\hline $2008-2012$ & 0.945 & -0.161 & 2.050 & 2.039 & 1.972 & 2.107 & 0.9549 & 0.4519 & 1.585 & 1.516 & 1.654 & 0.4536 \\
\hline $2008-2015$ & 0.918 & 0.134 & 1.701 & 2.046 & 1.997 & 2.096 & 0.9605 & 0.4244 & 1.657 & 1.606 & 1.707 & 0.4271 \\
\hline $2008-2016$ & 0.702 & -0.004 & 1.409 & 2.06 & 2.015 & 2.105 & 0.9634 & 0.4091 & 1.648 & 1.602 & 1.694 & 0.4136 \\
\hline
\end{tabular}

RMSE represents root-mean-square error; $R^{2}$ represents the coefficient of determination for the first linear equation. 